\title{
Predictive value of a self-reported history of varicella infection in determining immunity in adults
}

\author{
Candice N. Holmes, Karl T. Iglar, Brenda J. McDowell, Richard H. Glazier
}

\section{Abstract}

THE RECENT INTRODUCTION OF A VACCINE FOR VARICELLA has raised questions about whether, for adults, a patient's history of varicella infection is useful in determining if vaccination is necessary. We report findings on 184 family medicine patients aged 18 to 65 years who were asked if they had a history of varicella infection and were subsequently tested for varicella antibodies. A history of infection was positive for 114 (62\%) of the participants and negative or uncertain for 70 (38\%). All 114 subjects who reported a varicella infection history were immune. All 4 subjects who were not immune reported an uncertain or negative infection history. Except for people who are at increased risk of varicella infection or complications from infection, serologic testing may not be required for adults in the general population who have a history of varicella infection.

CMAJ 2004;171(10):1195-6

$\mathrm{V}$ aricella infection (chickenpox) is highly contagious ${ }^{1}$ and is spread by respiratory droplets or direct contact. Varicella can occur in nonimmune adults, in whom severity of the infection increases with age, ${ }^{2}$ often causing serious morbidity and loss of work time. In Canada, $70 \%$ of 53 reported deaths caused by varicella between 1987 and 1996 occurred in adolescents and adults. ${ }^{3}$ When acquired during pregnancy, varicella can cause significant maternal, perinatal and infant morbidity. ${ }^{3}$ After varicella infection, more than $95 \%$ of people develop antibodies, ${ }^{4}$ which are detected by serologic testing and indicate lifelong immunity.

The recent introduction of a vaccine for varicella means that nonimmune adults may benefit from vaccination. ${ }^{5}$ This has led to studies to determine whether a self-reported history of varicella infection is an accurate indication of the presence of antibodies. Studies to date have yielded disparate conclusions and recommendations, ${ }^{6-11}$ and no study has yet investigated this question for adults in a primary care population. Our objective was to determine, in an adult primary care population, the accuracy of a self-reported history of varicella infection in determining immunity.

St. Michael's Hospital Family Practice Unit serves a population with a wide range of socioeconomic groups and countries of origin. The mean patient age is 44 years, and $60 \%$ of patients are female. On randomly selected clinic dates between October and December 2000 we enrolled patients aged 18 to 65 years who were having blood taken for reasons other than serologic testing for varicella antibodies. Using a structured interview, patients were asked about their history of varicella infection (Box 1), and their blood samples were analyzed for antibody titres using the VZVscan latex agglutination test (Becton Dickinson, Cockeysville, Md.). We excluded patients with psychiatric or medical conditions impairing memory, a history of varicella vaccination or active varicella infection. All patients gave informed consent, and the study was approved by the St. Michael's Hospital Research Ethics Board.

A sample size of 200 patients provides power to detect a $95 \%$ confidence interval (CI) of $\pm 3 \%$ if the baseline positive predictive value of a history of varicella is $95 \%$.

Of 204 participants enrolled, 184 had a serologic test for varicella antibodies. For unknown reasons, the serologic test was not performed in the remaining 20 cases.

Of the 184 participants whose blood sample was tested for varicella antibodies, 101 (55\%) were women, 117 (64\%) were born in North America, and 107 (58\%) had postsecondary education. The mean age was 43 (standard devia-

Box 1: Clinical interview questions

- What is your age, highest level of schooling and country of birth?

- Do you have a personal history of any medical or psychiatric disorder that impairs your memory, perception or cognition?

- Are you taking any medication or other substance that impairs your memory, perception or cognition?

- Have you ever received the varicella vaccine (the vaccine for chickenpox)?

- Do you currently have chickenpox or shingles?

- Have you ever had chickenpox? At what age?

- Have you ever had shingles? At what age? 
tion 12.8) years. Participants for whom serologic testing was not done had similar characteristics.

All 114 (62\%) of the subjects who reported a history of varicella infection were immune, for a positive predictive value of $100 \%$ (95\% CI $97 \%-100 \%$ ) (Table 1). Of the 70 patients $(38 \%)$ who were uncertain of or reported no previous varicella infection, $4(6 \%)$ were not immune. The age of these 4 subjects ranged from 30 to 45 years, and 3 of the 4 were women who were born outside of North America.

In this study, a self-reported history of varicella infection was a highly accurate indicator of immunity to the pathogen. The 98\% (180/184) seroprevalence of varicella-zoster virus antibodies in our study population was slightly higher than other population estimates, ${ }^{2}$ and consequently a large majority of those who were uncertain of or reported no past infection were also immune.

Although we recruited participants from only 1 family medicine clinic in an urban teaching centre, the patient population of the clinic is diverse in terms of patient country of origin, socioeconomic status and demographic characteristics.

Whether particular vaccination strategies are appropriate or cost-effective depends on the population examined and their circumstances. The costs of serologic testing and vaccination and the potential financial, social and medical consequences of infection should be considered.

For people who are at increased risk of varicella infection or for whom it is crucial to establish immunity, such as health care workers, ${ }^{9,10,12,13}$ pregnant women ${ }^{7,11,14}$ and household contacts of immunocompromised people, it may be prudent to have them undergo routine serologic testing regardless of their self-reported infection history.

In this primary care setting, a positive history of varicella

\begin{tabular}{|c|c|c|c|}
\hline \multirow{2}{*}{$\begin{array}{l}\text { History of } \\
\text { varicella infection }\end{array}$} & \multicolumn{2}{|c|}{$\begin{array}{l}\text { Serologic } \\
\text { immune status, } \\
\text { no. of patients }\end{array}$} & \multirow[b]{2}{*}{ Total } \\
\hline & Yes & No & \\
\hline Yes & 114 & 0 & 114 \\
\hline No & 66 & 4 & 70 \\
\hline Total & 180 & 4 & 184 \\
\hline \multicolumn{4}{|c|}{$\begin{array}{l}\text { *Sensitivity: } 114 / 180=63 \%(95 \% \text { confidence interval [Cl] } 56 \%- \\
70 \% \text { ) specificity: } 4 / 4=100 \%(95 \% \mathrm{Cl} 40 \%-100 \%) \text {; positive } \\
\text { predictive value: } 114 / 114=100 \%(95 \% \mathrm{Cl} 97 \%-100 \%) \text {. Exact } \\
\text { confidence intervals were calculated on the basis of the binomial } \\
\text { distribution. }\end{array}$} \\
\hline
\end{tabular}

infection was an accurate indicator of the presence of antibodies. Except for people at high risk of varicella infection, serologic testing may not be required for adults in the general population who have had the infection. Vaccination should be offered to nonimmune patients.

This article has been peer reviewed.

From the Departments of Family and Community Medicine, University of Toronto and St. Michael's Hospital (Holmes, Iglar, McDowell, Glazier), and the Inner City Health Research Unit, St. Michael's Hospital (Glazier), Toronto, Ont.

Competing interests: None declared.

Contributors: Candice Holmes contributed to all stages of the research, including the study conception and design and the data acquisition, analysis and interpretation, and wrote the article. Karl Iglar contributed to the study conception and design and to the data acquisition. Brenda McDowell contributed to the study conception and design. Richard Glazier contributed to the data analysis and interpretation. All authors contributed to revising the article and gave final approval of the final submitted version.

Acknowledgements: Serologic testing was performed by St. Michael's Hospital Microbiology Laboratory. The serologic testing was financed by Glaxo Wellcome Canada Inc. Glaxo Wellcome Canada played no other role in this article, including study design, data acquisition, analysis or interpretation or the decision to publish study results.

\section{References}

1. US Centers for Disease Control and Prevention. Prevention of varicella: recommendations of the Advisory Committee on Immunization Practices (ACIP). MMWR Morb Mortal Wkly Rep 1996;45(RR-11):1-36.

2. Institute for Clinical Evaluative Sciences. A disease most fowl: an update on the varicella-zoster virus. ICES 1999;5(4):1,3,8.

3. National Advisory Committee on Immunization. Statement on recommended use of varicella virus vaccine. Can Comm Dis Rep 1999;25(ACS-1).

4. Glantz JC, Mushlin AI. Cost-effectiveness of routine antenatal varicella screening. Obstet Gynecol 1998;91:519-28.

5. Hoey J. Varicella vaccine update: Need for a booster? CMA7 2003;168(5):589.

6. Meurer LN, Slawson JG. Varicella vaccination strategies. F Fam Pract 1998; 47(5):331-2.

7. Silverman NS, Ewing SH, Todi N, Montgomery OC. Maternal varicella history as a predictor of varicella immune status. 7 Perinatol 1996;16:35-8.

8. Alagappan K, Fu L, Strater S, Atreidis V, Auerbach C. Seroprevalence of varicella antibodies among new house officers. Ann Emerg Med 1999;33(5):516-9.

9. Gallagher J, Quaid B, Cryan B. Susceptibility to varicella zoster virus infection in health care workers. Occup Med 1996;46:289-92.

10. Karunajeewa HA, Kelly HA. Predictive value of personal recall of chickenpox infection: implications for the use of varicella vaccine. Med 7 Aust 2001;174:153

11. Burnham B, Wells T. A cost-benefit analysis of a routine varicella vaccination program for United States Air Force Academy cadets. Mil Med 1998;163:631-4.

12. Coyle PV, McCaughey C, Wyatt DE, O'Neill HJ. Varicella vaccine in pregnancy: testing should be offered to women without a history of chickenpox. BMf 1997;314:226.

13. Nordin J, Baken L, Carlson R, Hering J. Age-specific rates of serological immunity in patients with a negative history for varicella infection [letter]. Infect Control Hosp Epidemiol 1998;19:823-4.

14. Enders G, Miller E, Cradock-Watson J, Bolley I, Ridehalgh M. Consequences of varicella and herpes zoster in pregnancy: prospective study of 1739 cases. Lancet 1994;343(8912):1548-51.

Correspondence to: Dr. Candice Holmes, St. Michael's Hospital, 30 Bond St., Toronto ON M5B 1W8; fax 416 867-7498 